\title{
Mechanical analysis of the dry stone walls built by the Incas
}

\author{
Jaime Castro ${ }^{1, \star}$, Luis E. Vallejo ${ }^{2, \star \star}$, and Nicolas Estrada ${ }^{1, \star \star \star}$ \\ ${ }^{1}$ Departamento de Ingeniería Civil y Ambiental, Universidad de los Andes, Bogotá, Colombia \\ ${ }^{2}$ Department of Civil and Environmental Engineering, University of Pittsburgh, Pittsburgh PA, USA
}

\begin{abstract}
In this paper, the retaining walls in the agricultural terraces built by the Incas are analyzed from a mechanical point of view. In order to do so, ten different walls from the Lower Agricultural Sector of Machu Picchu, Perú, were selected using images from Google Street View and Google Earth Pro. Then, these walls were digitalized and their mechanical stability was evaluated. Firstly, it was found that these retaining walls are characterized by two distinctive features: disorder and a block size distribution with a large size span, i.e., the particle size varies from blocks that can be carried by one person to large blocks weighing several tons. Secondly, it was found that, thanks to the large span of the block size distribution, the factor of safety of the Inca retaining walls is remarkably close to those that are recommended in modern geotechnical design standards. This suggests that these structures were not only functional but also highly optimized, probably as a result of a careful trial and error procedure.
\end{abstract}

\section{Introduction}

The Inca Empire was the largest pre-Columbian civilization of the American continent, with a surface close to one million square kilometres and a population close to twelve million people during the sixteenth century. Their development relied on a remarkable infrastructure network of bridges, roads, agricultural terraces, buildings, and water conduction systems. Most of these structures were built with stone blocks, more than 500 years ago. Yet, many of them remain in good conditions without any significant maintenance, demonstrating a noteworthy engineering expertise.

Amongst the different types of structures built by the Incas, one of the most important was the system of agricultural terraces. These terraces were an essential part of the Inca food supply system [1], allowing the development of a variety of crops throughout the Andes, especially maize, potatoes, and quinoa.

Despite their importance and engineering splendor, Inca agricultural terraces have been seldom studied from an engineering perspective. The works of Wright and Valencia-Zergara must be noted [2-5], in which water management and drainage in terraces of Machu Picchu, Tipon, and Moray were analyzed. Soil composition and stratification were then studied by Wright and Dr. James Self [6]. However, investigations in which these systems are analyzed from a mechanical point of view remain scarce. Probably, the first researcher to carry out this kind of analyses was Vallejo in 2014 [7].

\footnotetext{
$\star$ e-mail: jj.castro429@uniandes.edu.co

$\star \star$ e-mail: vallejo@pitt.edu

$\star \star \star$ e-mail: n.estrada22@uniandes.edu.co
}

The purpose of this work was to analyze the mechanical stability of the retaining walls used in the agricultural terraces built by the Incas. This was carried out by means of a careful image analysis coupled with a simple mechanical model, which allowed for highlighting the functionality and optimality of these retaining walls, even while built by means of a trial and error procedure.

In the following, the location and description of the analyzed walls are presented in Secs. 2 and 3. The procedures followed for analyzing the mechanical stability of these systems is presented in Sec. 4, and the paper ends in Sec. 5 with a brief conclusion.

\section{Location of the analyzed walls}

In order to carry out this investigation, ten retaining walls were selected from the Lower Agricultural Sector of Machu Picchu, Perú. These walls were selected by analyzing simultaneously images from Google Street View and Google Earth Pro. The images from Google Street View were necessary to identify the blocks that compose each of the walls, and the images from Google Earth Pro were used to define the length scale. Figure 1 shows the location of nine of the analyzed walls.

\section{Description of the analyzed walls}

As a first step, the retaining walls were qualitatively analyzed and compared to masonry walls used in other type of Inca structures. Figures 2 (a) and (c) show pictures of two walls. The first one corresponds to wall 6 in Fig. 1 (i.e., a wall used in an agricultural terrace), and the second one is a typical masonry wall used in a house. Figures 


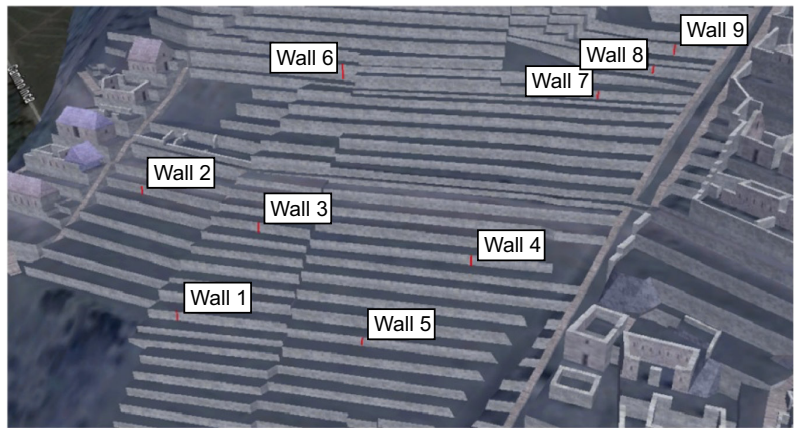

Figure 1. Location of nine of the analyzed walls in a Google Earth Pro image. The walls height, indicated by the red lines, varies from 1.58 to $3.40 \mathrm{~m}$, with a average value of $2.02 \mathrm{~m}$.

2 (b) and (d) show the result of a DEM simulation of the same systems ${ }^{1}$. The red lines represent the normal forces between particles, the thickness being proportional to the force intensity.

The microstructure observed in both types of walls (i.e., retaining wall and masonry wall) is notably different. The retaining wall is characterized by a disordered arrangement, made up of blocks of different shapes and sizes. The size of these blocks varies from $4 \mathrm{~cm}$ up to $1.5 \mathrm{~m}$, and the mass varies approximately from $100 \mathrm{~g}$ up to 7 tons, which must have implied serious construction difficulties. On the other hand, the masonry wall is characterized by an ordered arrangement, formed by blocks with similar shape and size, comparable to a modern-day masonry wall. The blocks in the masonry wall have a manageable size, so that they can be easily carried and placed by one person, which is very practical from a construction point of view.

In order to compare the block size distributions of the different walls, it is convenient to analyze the cumulate mass distributions, also known as the material's grading curves. Figure 3 shows the grading curves for the ten analyzed retaining walls, as well as for two masonry walls (i.e., walls 11 and 12). It can be seen that the grading curves of the retaining walls cover a much broader range of sizes, while the grading curves of masonry walls are basically uniform (i.e., all blocks have approximately the same size). The degree of uniformity of the block size distributions can be quantified by means of the uniformity coefficient $C_{u}=D_{60} / D_{10}$, where $D_{60}$ is the diameter of the block for which $60 \%$ of the blocks are smaller, and $D_{10}$ is the diameter of the block for which $10 \%$ of the blocks are smaller. For the analyzed retaining walls, $C_{u}$ varied from 1.80 to 4.41 , with an average value of 2.76 . For the masonry walls, the average $C_{u}$ was 1.58 . This confirms that the retaining walls are characterized by a block size distribution with a large size span, whereas the masonry walls used in buildings are characterized by a much narrower range of sizes.

\footnotetext{
${ }^{1}$ Simulations were carried out using the LMGC90 software, developed in Montpellier, which is well suited for simulating large assemblies of rigid grains of arbitrary shape.
}
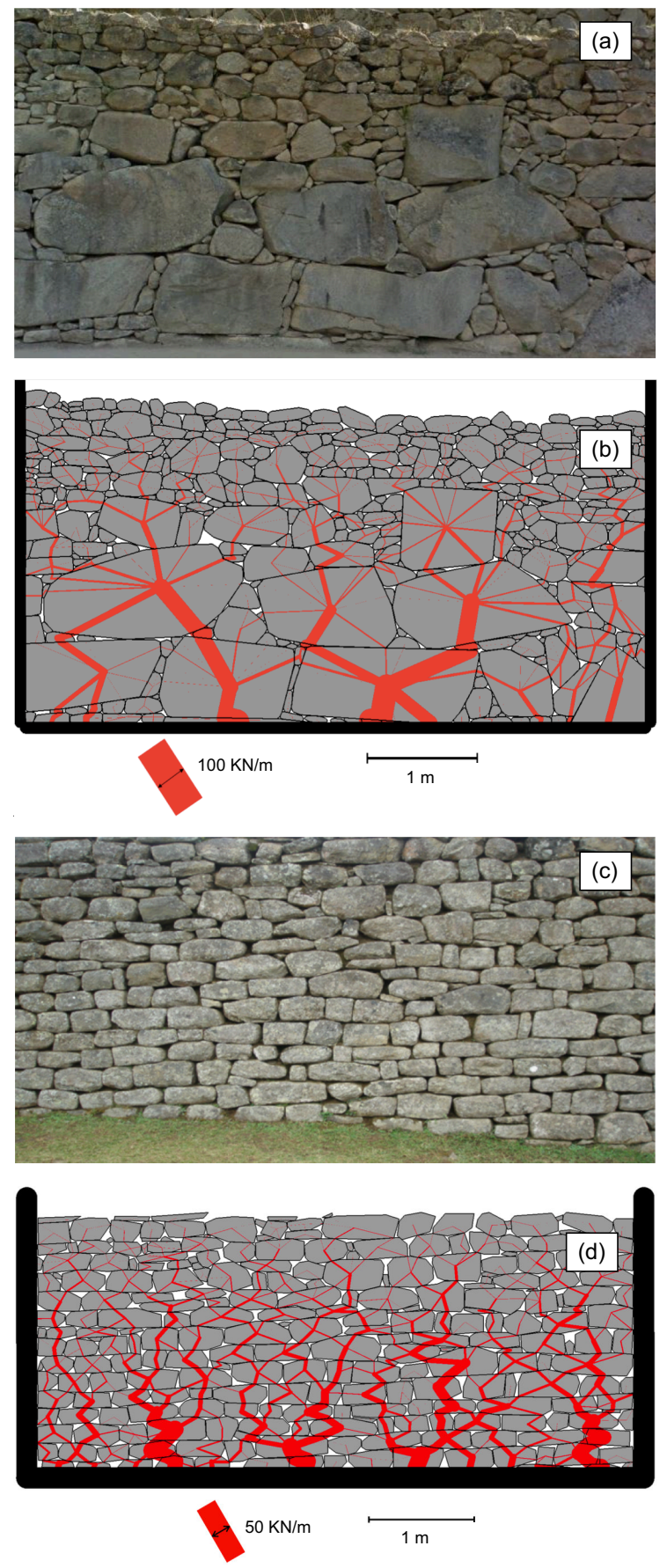

Figure 2. Picture (a) and DEM simulation (b) of a retaining wall in an agricultural terrace (i.e., wall 6 in Fig. 1). Picture (c) and DEM simulation (d) of a masonry wall in a house.

\section{Stability of the retaining walls}

The mechanical stability of the analyzed retaining walls was assessed by means of the safety factor against sliding and overturning. The initial necessary step was to identify the mechanical parameters of the soil and the rock blocks. Most of these values must be assumed, since it is not possible to extract soil samples in order to conduct laboratory tests, because of the archeological importance of these systems. The analyses presented in this paper were based on the values suggested in [8]. The unit weight of the rock 


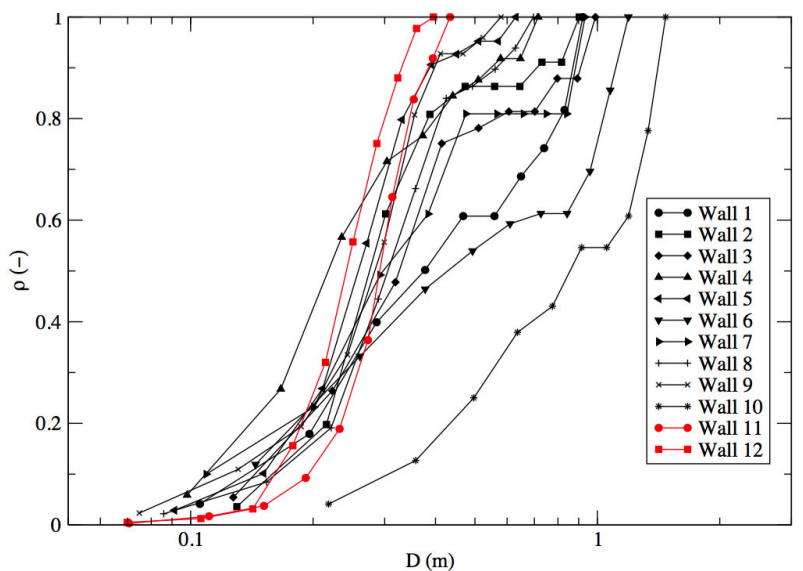

Figure 3. Grading curves for ten analyzed retaining walls used in agricultural terraces (i.e., walls 1 to 10 ) and for two masonry walls (i.e., walls 11 and 12).
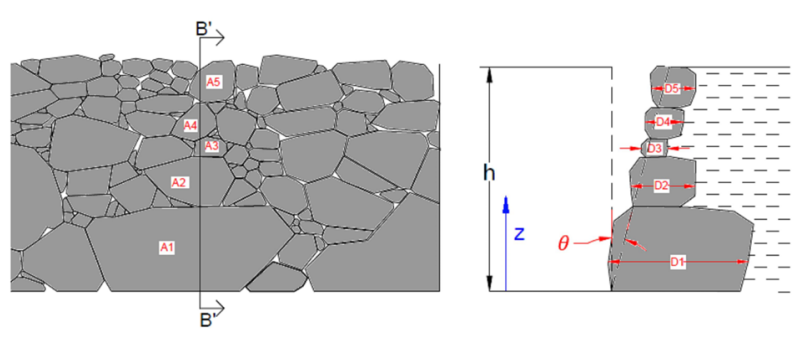

Figure 4. Left: Schematic representation of a retaining wall. Right: Internal structure assumed for section B'B'.

blocks was $\gamma_{b}=25.36 \mathrm{KN} / \mathrm{m}^{3}$, and the dry unit weight of the soil was $\gamma_{s}=15.71 \mathrm{KN} / \mathrm{m}^{3}$. The friction angle between blocks was $\phi_{b} \in\left(33^{\circ}, 38^{\circ}\right)$, and the soil's internal friction angle was $\phi_{s} \in\left(20^{\circ}, 30^{\circ}\right)$.

The next step was to assume the structure of the wall inside the terrace. In order to to this, the block's area $A$ in the face of the wall (i.e., the area of the particles shown in Figs. 2(b) and (d)) was measured, and the diameter $D=$ $\sqrt{4 A / \pi}$ of a circle having the same area was calculated. Then, it was supposed that the depth of the particles in the direction perpendicular to the face of the wall was $D$. This structure is shown schematically in Fig. 4. Figure 4 also shows the inclination $\theta$ of the wall towards the terrace, which was assumed in the range $\left(7^{\circ}, 15^{\circ}\right)$ as reported in [9], as well as the total height $h$ and the height $z$.

The final step was to calculate the factor of safety $S F$ for two possible failure modes: sliding ${ }_{s}$ and overturning $o$.

For the sliding failure mode, $S F_{S}$ was calculated as $f_{r} / f_{d}$, where $f_{r}$ is the resisting force and $f_{d}$ is the driving force ${ }^{2}$. When evaluating a possible failure plane at any height $z, f_{r}$ is the friction force between the portion of the wall above $z$ and that below $z . f_{d}$ is the force applied by the soil on the portion of the wall above $z$, which was calculated with the Rankine theory, by considering a

\footnotetext{
${ }^{2} S F>1$ corresponds to a stable system and $S F<1$ to an unstable system.
}

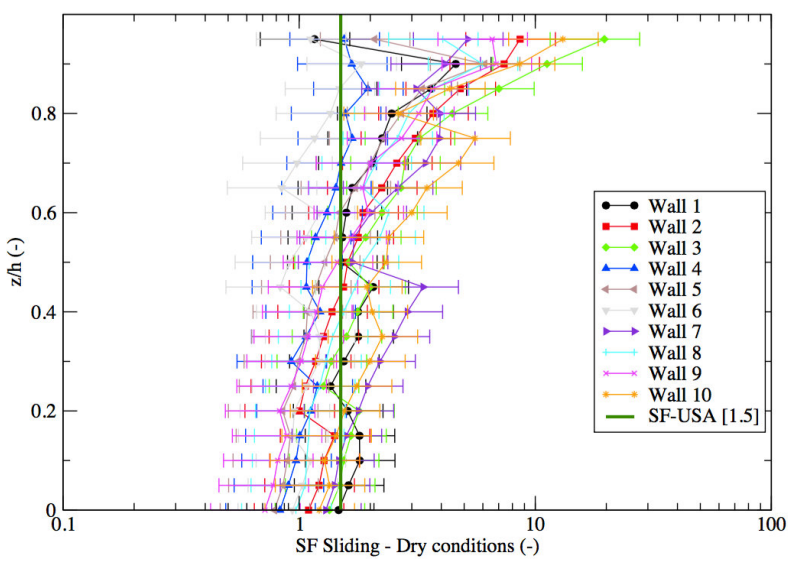

Figure 5. Factor of safety $S F_{s}$ for the sliding failure mode as a function of the normalized height $z / h$. The green line at $S F_{s}=1.5$ indicates the factor of safety recommended in modern geotechnical design standards in USA. Solid symbols indicate the average value and error bars indicate the maximal and minimal values.

dry soil. This condition is appropriate since the walls were built without any mortar to bind the blocks together, ensuring that the walls behave as drained systems. $S F_{s}$ is then given by

$$
S F_{s}=\frac{\sum\left(\gamma_{b} A_{i} D_{i}\right) \tan \phi_{b}}{K \gamma_{s}(h-z)^{2} / 2},
$$

where the sum runs over all blocks with center of gravity above $z, A_{i}$ and $D_{i}$ are the area and the diameter of block $_{i}$, respectively, and $K$ is the coefficient of lateral earth pressure, which was varied between the active earth pressure $\left(1-\sin \phi_{s}\right) /\left(1+\sin \phi_{s}\right)$ and the at-rest earth pressure $1-\sin \phi_{s}$.

Figure 5 shows $S F_{s}$ as a function of the normalized height $z / h$ for all analyzed walls. Firstly, it can be seen that $S F_{S}$ changes with $z / h$, being larger for the top portion of the walls and smaller at the base of the walls. This is to be expected, since $f_{r}$ increases linearly with depth (i.e., with $h-z$ ) while $f_{d}$ increases as $(h-z)^{2}$. Thus, the walls must necessarily become unstable at a certain depth $h-z$. Secondly, it can be seen that the evolution of $S F_{S}$ with $z / h$ is not gradual, but exhibits strong fluctuations. These fluctuations are due to particularly large blocks, which drastically increase $W_{b}$ and, thus, $f_{r}$. This effect is important, because it allows for building higher walls than those that could be built using only medium-sized blocks, such as those used in masonry walls. Thirdly, it is remarkable that $S F_{s}$ in most of the walls' height is close to 1.5 (indicated by the vertical line in the figure). This is the factor of safety recommended in modern geotechnical design standards in North America [10-12]. Finally, it can be observed that $S F_{S}$ at the base of the walls is close to unity, evidencing a highly optimized design, even while reached by means of a trial and error procedure.

For the overturning failure mode, $S F_{o}$ was calculated as $M_{r} / M_{d}$, where $M_{r}$ is the resisting moment and $M_{d}$ is the driving moment, with respect to a point $o^{\prime}$ located at the face of the wall. When evaluating a possible failure at 


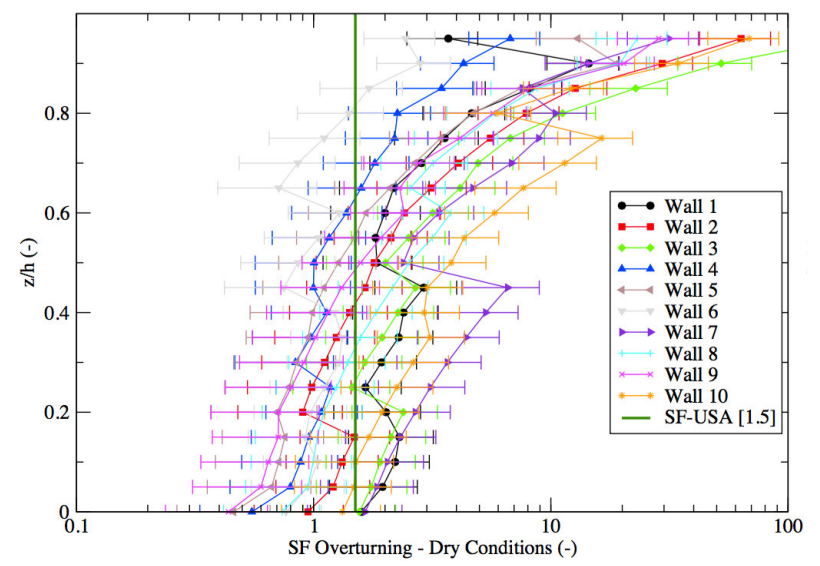

Figure 6. Factor of safety $S F_{o}$ for the overturning failure mode as a function of the normalized height $z / h$. The green line at $S F_{o}=1.5$ indicates the factor of safety recommended in modern geotechnical design standards in USA. Solid symbols indicate the average value and error bars indicate the maximal and minimal values.

any height $z, M_{r}$ is the moment exerted by the weight of the blocks above $z$ with respect to $o^{\prime}$. $M_{d}$ is the moment of the force applied by the soil on the portion of the wall above $z$ with respect to $o^{\prime} . S F_{o}$ is then given by

$$
S F_{o}=\frac{\sum\left[\left(\gamma_{b} A_{i} D_{i}\right)\left(D_{i} / 2+\left(z_{i}-z\right) \tan \theta\right]\right.}{K \gamma_{s}(h-z)^{3} / 6},
$$

where the sum runs over all blocks with center of gravity above $z$ and $\theta$ is the inclination of the face of the wall.

Figure 6 shows $S F_{o}$ as a function of the normalized height $z / h$ for all analyzed walls. As for $S F_{s}$, it can be seen that large blocks play an important role in keeping $S F_{o}$ above unity, $S F_{o}$ for most of the walls' height is close to 1.5 , and $S F_{o} \simeq 1$ at the base of the walls, evidencing the optimal design of these retaining structures.

\section{Conclusion}

The purpose of this work was to analyze the mechanical stability of the retaining walls used in the agricultural terraces built by the Incas. For so doing, ten retaining walls from the Lower Agricultural Sector of Machu Picchu were selected and their mechanical stability was analyzed. In order to illustrate these analyses, the structure of the retaining walls was also analyzed and compared with that of masonry walls used in houses and temples.

Firstly, our results show that retaining walls used in terraces have a characteristic microstructure that is significantly different from those observed in masonry walls. Specifically, when building retaining walls, the Incas avoided ordered patterns and used a block size distribution with a large size span (i.e., the blocks size varies from blocks that can be carried by one person to large blocks weighing several tons).

Secondly, the mechanical stability of the retaining walls was analyzed by estimating the factor of safety, considering two possible failure mechanisms: sliding and overturning, as it is usually done for geotechnical retaining structures. It was shown that, thanks to the large size span of the block size distribution, the factor of safety fluctuates inside a range of values that are remarkably close to those that are recommended in modern geotechnical design standards. It is also shown that the stability condition at the base of the walls is close to limit equilibrium, as long as the system is considered drained. This could explain why the Incas did not use any mortar to bind the blocks together, allowing the phreatic water to drain freely.

Finally, our conclusions suggest that Inca retaining walls are not only functional but also highly optimized, probably as a result of a careful trial and error design procedure.

\section{Acknowledgements}

We thank Jaime Wills and Luisa Orozco for fruitful discussions, and Emilien Azéma for his assistance with the LMGC90 platform.

\section{References}

[1] C. Graber, Smithsonian Magazine (2011)

[2] K. Wright, Journal of Hydraulic Engineering (1997)

[3] K. Wright, A. Valencia Zegarra, C.M. Crowley, Tech. rep., Instituto Nacional de Cultura del Perú, Wright Paleohydrological Institue, Wright Water Engineers (2000)

[4] K. Wright, A. Valencia Zegarra, R.M. Wright, G. McEwan, Tech. rep., Instituto Nacional de Cultura del Perú, Wright Paleohydrological Institue, Wright Water Engineers (2001)

[5] K. Wright, Water environment and technology pp. 78-87 (2008)

[6] K. Wright, A. Valencia Zegarra, W.L. Lorah, Journal of Irrigation and Drainage Engineering 125 (1999)

[7] L.E. Vallejo, M. Fontanese, Geo-Congress 2014 Technical Papers (2014)

[8] T.R. West, Geology Applied to Engineering (Waveland Press, Long Grove, Illinois, 2010)

[9] T.G.S. of London, J. Dixon, Geoscientist (December 2014)

[10] E.E.C. Ltd., Tech. rep., City of Nanaimo (2014)

[11] C.D. of Transportation, Tech. rep., State of California (2004)

[12] D. of Public Works-Building, S. Division, Tech. rep., County of Los Angeles (2011) 